\title{
CTMC calculations of electron capture and ionization in collisions of multiply charged ions with elliptical Rydberg atoms
}

\author{
J. Lu ${ }^{1}$, Z. Roller-Lutz ${ }^{2}$, H.O. Lutz ${ }^{1}$ \\ ${ }^{1}$ Fakultät für Physik, Universität Bielefeld, 33501 Bielefeld, Germany \\ ${ }^{2}$ Institute of Physics, Faculty of Medicine, Rijeka University, Rijeka, Croatia
}

\begin{abstract}
We have performed classical trajectory Monte Carlo (CTMC) studies of electron capture and ionization in multiply charged $(Q \leq 8)$ ion-Rydberg atom collisions at intermediate impact velocities. Impact parallel to the minor and to the major axis, respectively, of the initial Kepler electron ellipse has been investigated. The important role of the initial electron momentum distribution found for singly charged ion impact is strongly disminished for higher projectile charge, while the initial spatial distribution remains important for all values of $\mathrm{Q}$ studied.
\end{abstract}

The use of coherent elliptical Rydberg states in ion-atom collision studies (for recent papers on the subject cf. [1-3] and references therein) has not only aided the intuitive understanding of the interaction dynamics, it also illuminates the roles of the momentum and the spatial distributions of the target electron states. In classical terms, the momentum distribution can be widely varied simply by changing the eccentricity $\varepsilon$ of the Rydberg ellipse without affecting the energy of the state. In particular, for impact perpendicular to the major axis of the ellipse, the capture cross section displays a maximum if $\mathbf{v}_{\mathbf{p}}$ (the perihelion electron velocity) is parallel and equal to the projectile velocity $\mathbf{v}$; this is believed to be due to the matching electron momenta in the initial target and the final projectile state. In contrast, the role of the spatial distribution becomes most clearly visible if the impact velocity vector in adjusted perpendicular to the minor axis of the Rydberg ellipse; in this case, the electrons can be located either between the approaching ion and the target nucleus ("upstream geometry") or behind the target nucleus, as seen from the projectile ("downstream geometry") without otherwise changing the momentum distribution of the Rydberg state (i.e., its angular momentum $l$ and the principal quantum number $n$ ). The capture cross section in both cases turns out to be quite different: it is much larger in the upstream case as compared to the downstream case; apparently, in the corresponding region of parameter space the spatial characteristics of the initial state determine the outcome of the collision. These investigations have so far been restricted to collisions with singly charged ions. Recently, however, it has become possible to employ such targets in studies involving multiply charged ions [1,4]. In another context (electron capture by multiply charged ion in the presence of an external magnetic field) we have found indications [5] that for increasing projectile charge $\mathrm{Q}$ the dis- 
tortion of the initial state increasingly dominates over the influence of different target electron distributions; we have therefore performed an exploratory study of such systems which is the topic of this Letter.

We employ the classical trajectory Monte Carlo (CTMC) method which is quite useful in particular for the description of quantum mechanically complex systems, giving a good qualitative and often fairly quantitative agreement with experimental data ( for more recent applications to the study of Rydberg atom collisions cf., e.g., [1-3]). Structureless ions of charge Q between 1 and 8 collide with Rydberg target atoms with nuclear charge $\mathrm{q}=1$ and principal quantum number $n=25$. The geometry is chosen such that the direction of impact is perpendicular to the angular momentum direction of the Kepler ellipse. Specifically, two cases are studied: (i) the impact is parallel to the minor axis, thus allowing to study the velocity matching phenomenon, and (ii) impact parallel to the major axis, showing the effect of the spatial orientation of the target electron ("upstream-downstream asymmetry"). A useful quantity characterizing the electron orbit is the (generalized) eccentricity $\varepsilon= \pm \sqrt{1-(l / n)^{2}}$. In case (i), the + sign identifies orbits with the perihelion velocity $\mathbf{v}_{\mathbf{p}}$ parallel (-, antiparallel) to $\mathbf{v}$; in case (ii) it characterizes the upstream (-, downstream) geometry. The impact velocity $v$ is scaled by $1 / n$, the velocity of a circular Rydberg state, i.e., $V=v n=1$ in this case; the number of MC cycles was adjusted to obtain statistical uncertainties of less than 5\%. Care has been taken to assure that the projectile starts sufficiently far from the target (approximately $3.5 \times 10^{5}$ atomic units a.u.) to correctly describe the initial part of the trajectory; in view of the long range Coulomb potential and the known sensitivity of Rydberg states to $l, m$ changing processes, this is critical particularly for higher projectile charges $\mathrm{Q}$ (see also below).

\section{(i) Impact parallel to the minor axis}

Figure 1 shows the eccentricity-dependent charge capture cross section $\sigma / Q$ for $V=1.66$ and Q ranging from 1 to 8 . Velocity matching is obtained at $\varepsilon=+0.47$; at low $\mathrm{Q}$, the cross section displays the well-known behavior with a pronounced maximum at this eccentricity and a deep minimum for negative eccentricities which characterize a strongly elongated Kepler ellipse with $\mathbf{v}$ antiparallel to $\mathbf{v}_{\mathbf{p}}$. For increasing Q, this structure is soon washed out; while it is still noticeable for $\mathrm{Q}=2$ and 4 , almost any trace of the minimum has disappeared for $\mathrm{Q}=8$. Inspection of electron trajectories during the approach of the projectile ion reveals the reason: as expected, the long range Coulomb force distorts the original Kepler ellipse already at quite long distances. This distortion is quite regular, and reminds of a Stark effect. Indeed, a simple estimate confirms this: for $\mathrm{Q}=8$, an electric field strength of $5 \mathrm{~V} / \mathrm{cm}$ (i.e., of the order of the fields applied to the collision region in the experiments [1]) is attained at approximately $10^{5}$ a.u. This initial state effect might be reduced in the experiment by applying a strong field in the target region; however, this results also illuminates an inherent weakness of the CTMC-approach: the slow rise of the electric field may "in reality" induce adiabatic transitions between the many Rydberg states which would be populated differently in the classical calculation. This distortion of the initial state becomes quite severe at distances below $10^{4}$ a.u., i.e., corresponding to several revolution of the Rydberg electron about its nucleus; therefore, it is to be believed that the 
washing out of the cross section structure is indeed a real effect. Finally, we may add that also the impact parameter dependence of the capture probability reflects the signature of this effect. While for $\mathrm{Q}=1$ and $\varepsilon=+0.47$ (the velocity matching situation) the capture probability is rather concentrated about the perihelion position [6], it is nearly symmetric about $b=0$ (the position of the target nucleus) for $\mathrm{Q}=8$.

\section{(ii) Impact parallel to the major axis}

For this study, we choose $\varepsilon=0.96$, corresponding to $l=7$. This creates a quite elongated state which is not too non-classical (low $l$ ). Figure 2 shows for $\mathrm{Q}=8$ the cross sections for upstream $\left(\sigma_{u}\right)$ and downstream $\left(\sigma_{d}\right)$ geometry, respectively, including the contributions of the various "swaps" to the capture cross sections. A swap has been defined as a passage of the electron through the midplane between projectile and the target nucleus; note, however, that for asymmetric collisions $(q \neq Q$ as studied here), this plane has to cut the connection line between target and projectile nucleus at the saddle point of the two respective Coulomb potentials (i.e, at a distance $R /(1+\sqrt{q / Q})$ from the projectile, with $\mathrm{R}$ the distance of both nuclei). In view of the discussion in section (i) above, the strong upstreamdownstream asymmetry seen in these data is at first glance somewhat surprising. Again, inspection of the electron trajectories sheds light on this point: the slowly increasing electric field of the approaching projectile causes a distortion and precession of the Kepler ellipse, in general not strong enough, however, to revert the upstream into a downstream geometry and vice versa. Therefore, upon approach of the projectile into the actual close interaction the electron is still mainly fore or aft, respectively, of the target nucleus, thus qualitatively preserving the role of the initial spatial distribution of the electron; interestingly, even 3-swap and higher swap processes can still be discerned. The qualitatively different behavior of $\sigma_{u}$ and $\sigma_{d}$ beyond $V=1.5$ is associated with differently rising cross sections for ionization; in case of the downstream geometry it sets in at considerably smaller $\mathrm{V}$-values as compared to the upstream geometry. This is further clarified by the respective impact parameter dependencies (Fig.3a,b): In the upstream situation, charge exchange extends out to fairly large distances and ionization is still weak; in the downstream situation, the maximum charge exchange probability is of the same order as in the upstream case, however, it is limited to much smaller impact parameters, and ionization is already quite strong.

To conclude, our analysis shows that for impact of multiply charged ions of intermediate velocities the role of the initial electron momentum distribution becomes weaker for increasing projectile charge. This is due to the strong perturbation of the initial state by the approaching ion which induces pronounced changes in the momentum distribution long before the actual close interaction occurs. In contrast, the initial spatial orientation of the electron continues to be important for all Q-values studied here.

\section{Acknowledgment}

This work has been supported by the Deutsche Forschungsgemeinschaft (DFG). 


\section{References:}

[1 ] J. C. Day, B. D. DePaola, T. Ehrenreich, S. B. Hansen, E. HorsdalPedersen, Y. Leontiev and K. S. Mogensen 1997 Phys. Rev A56, 4700;

[2 ] D. M. Homan, O. P. Makarov, O. P. Sorokina, K. B. MacAdam, M. F. V. Lundsgaard, C. D. Lin, N. Toshima 1998, Phys.Rev A58, 4565 ;

[3 ] L. Kristensen, T. Bové, B. D. DePaola, T. Ehrenreich, E. HorsdalPerdersen and O. E. Povlsen submitted;

[4 ] B. D. DePaola, private communiation;

[5 ] J. Lu, S. Bradenbrink, Z. Roller-Lutz, and H.O. Lutz, 1999 J.Phys. B: At.Mol.Opt.Phys.32, L681;

[6 ] S. Bradenbrink, H. Reihl, Z. Roller-Lutz, and H.O. Lutz, 1995 J.Phys. B: At.Mol.Opt.Phys.28, L133; 


\section{Figure caption}

Fig. 1 Eccentricity-dependent capture cross section $\sigma / Q$ for impact velocity $V=$ 1.66 (in units of the circular $n=25$ Rydberg electron velocity) and different projectile charges Q. For the initial state, velocity matching is obtained at $\varepsilon=+0.47$.

Fig. 2 Charge capture cross sections for (a)upstream $\sigma_{u}$ and (b)downstream $\sigma_{d}$ geometry; the eccentricity $\varepsilon= \pm 0.96$. The respective ionization cross sections are also given.

Fig. 3 Impact parameter dependent probabilities of capture and ionization: (a) upstream geometry, (b) downstream geometry. Impact parameter $b$ in atomic units; impact velocity $V=1.5$; projectile charge $Q=8$. 


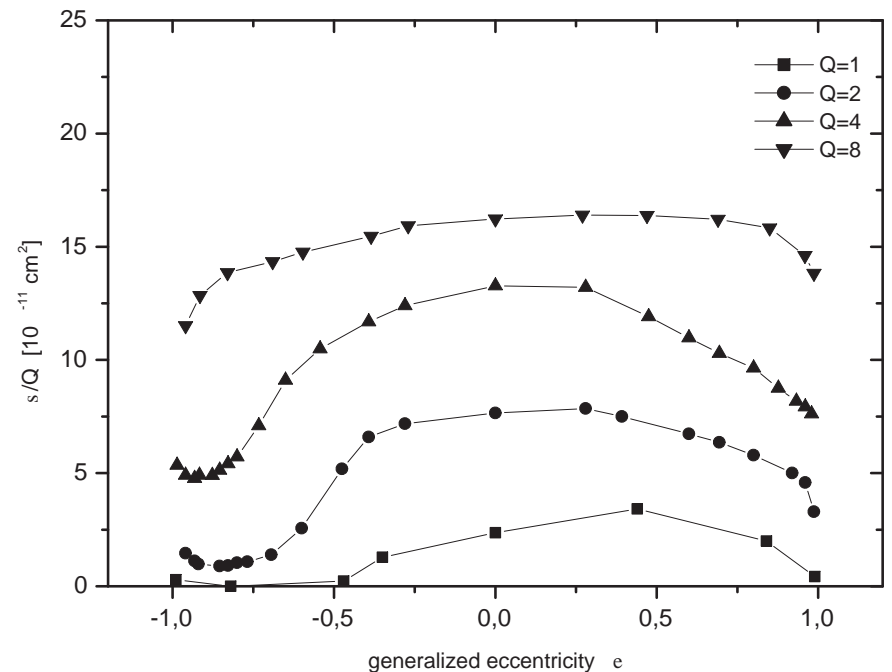




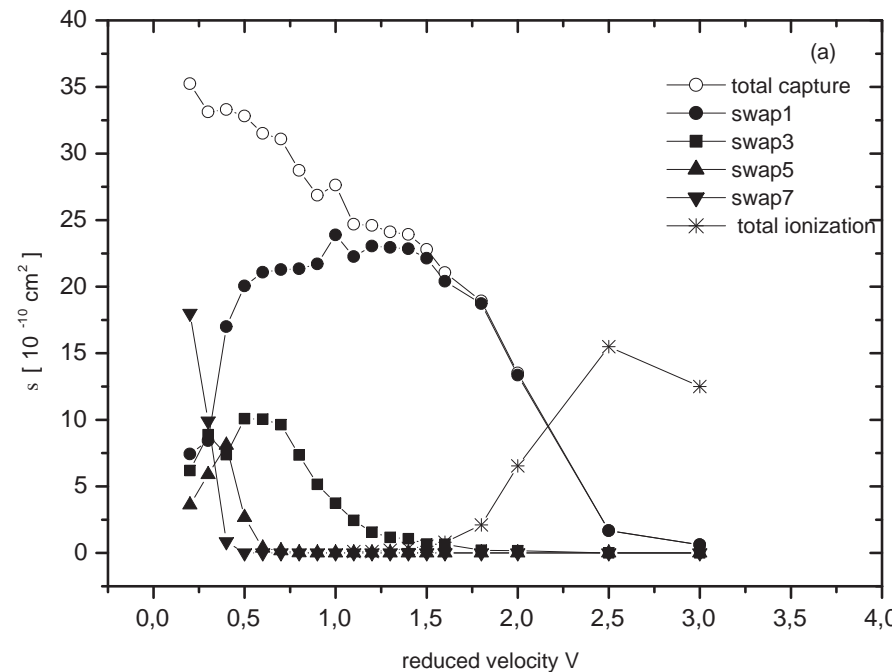




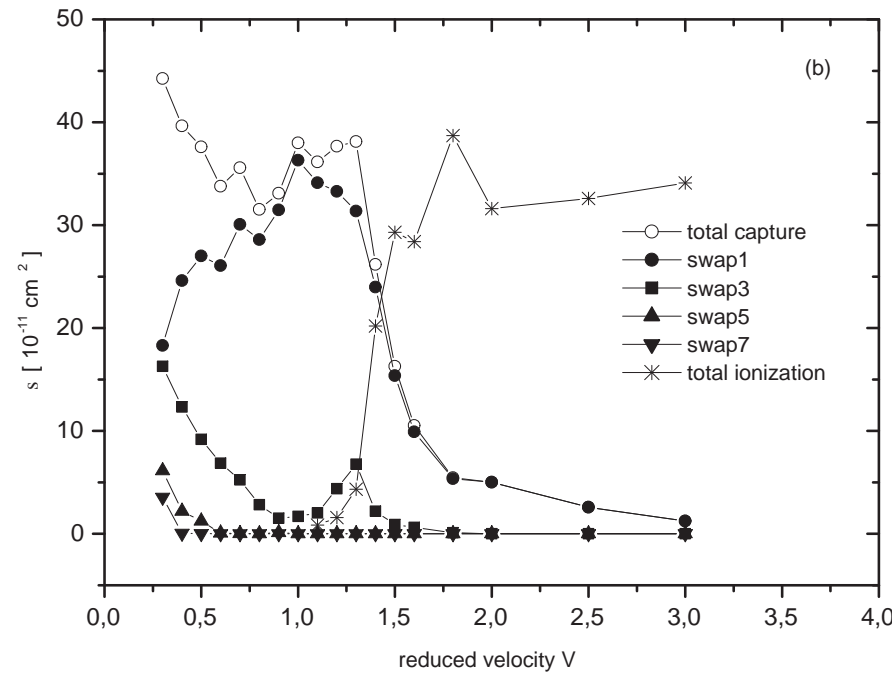




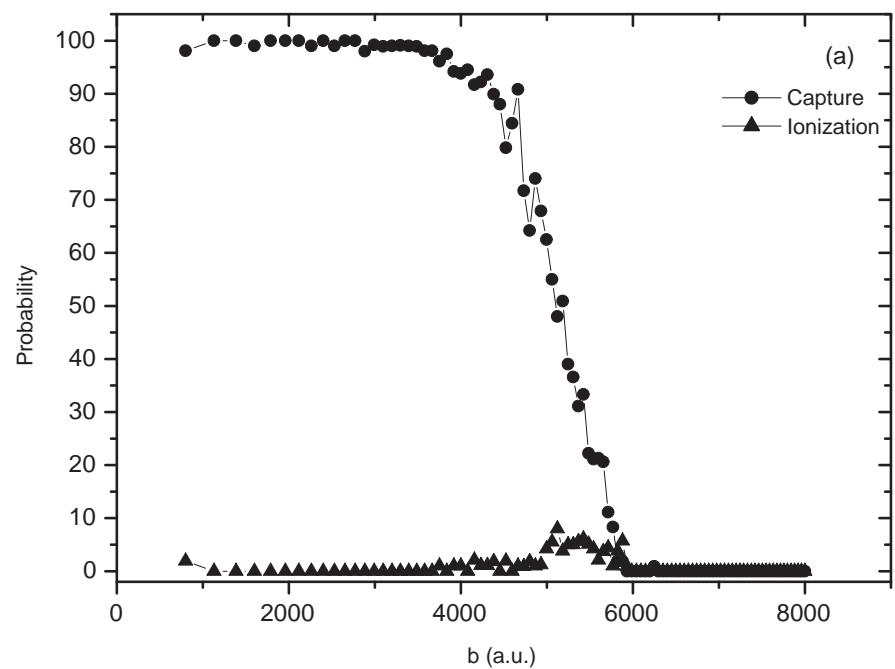




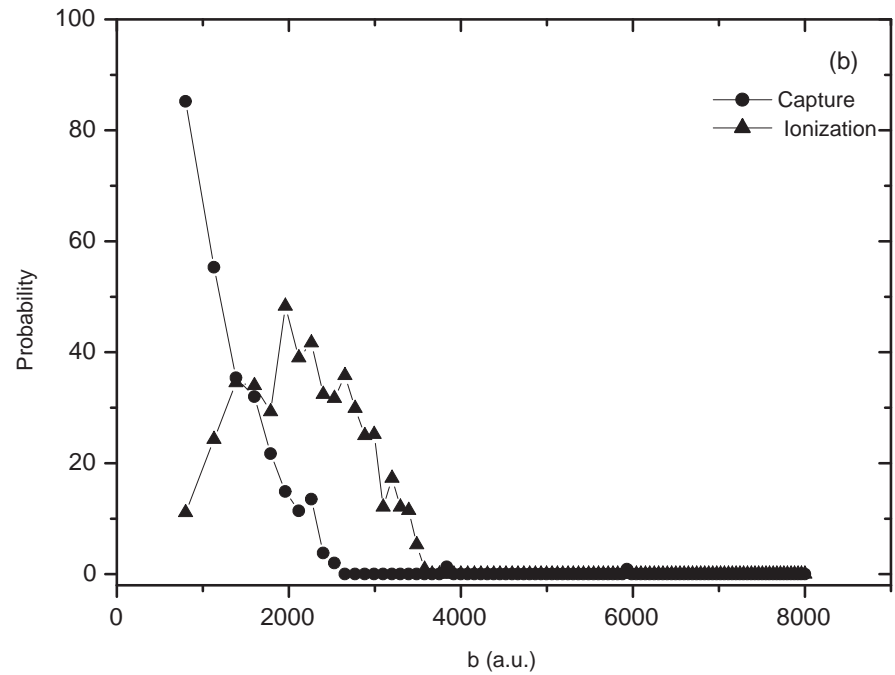

